GLASNIK MATEMATIČKI

Vol. 39(59)(2004), $303-314$

\title{
BOUNDED 2-LINEAR OPERATORS ON 2-NORMED SETS
}

\author{
ZOFIA LEWANDOWSKA \\ Pedagogical University, Poland
}

\begin{abstract}
In this paper properties of bounded 2-linear operators from a 2-normed set into a normed space are considered. The space of these operators is a Banach space and a symmetric 2-normed space. In the third part we will formulate Banach-Steinhaus Theorems for a family of bounded 2-linear operators from a 2-normed set into a Banach space.
\end{abstract}

\section{INTRODUCTION}

In [1] S. Gähler introduced the following definition of a 2-normed space:

Definition 1.1. [1] Let $X$ be a real linear space of dimension greater than 1 and let $\|\cdot, \cdot\|$ be a real valued function on $X \times X$ satisfying the following four properties:

(G1) $\|x, y\|=0$ if and only if the vectors $x$ and $y$ are linearly dependent;

(G2) $\|x, y\|=\|y, x\|$;

(G3) $\|x, \alpha y\|=|\alpha| \cdot\|x, y\|$ for every real number $\alpha$;

(G4) $\|x, y+z\| \leq\|x, y\|+\|x, z\|$ for every $x, y, z \in X$.

The function $\|\cdot, \cdot\|$ will be called a 2-norm on $X$ and the pair $(X,\|\cdot, \cdot\|)$ a linear 2-normed space.

In [4] and [5] we gave a generalization of the Gähler's 2-normed space. Namely a generalized 2-norm need not be symmetric and satisfy the first condition of the above definition.

Definition 1.2. [4] Let $X$ and $Y$ be real linear spaces. Denote by $\mathcal{D}$ a non-empty subset of $X \times Y$ such that for every $x \in X, y \in Y$ the sets

2000 Mathematics Subject Classification. 46A32,47B99.

Key words and phrases. 2-normed set, 2-normed space, bounded 2-linear operator, Banach-Steinhaus theorems. 
$\mathcal{D}_{x}=\{y \in Y ;(x, y) \in \mathcal{D}\}$ and $\mathcal{D}^{y}=\{x \in X ;(x, y) \in \mathcal{D}\}$ are linear subspaces of the space $Y$ and $X$, respectively.

A function $\|\cdot, \cdot\|: \mathcal{D} \rightarrow[0, \infty)$ will be called a generalized 2-norm on $\mathcal{D}$ if it satisfies the following conditions:

(N1) $\|x, \alpha y\|=|\alpha| \cdot\|x, y\|=\|\alpha x, y\|$ for any real number $\alpha$ and all $(x, y) \in$ $\mathcal{D}$;

(N2) $\|x, y+z\| \leq\|x, y\|+\|x, z\|$ for $x \in X, y, z \in Y$ such that $(x, y),(x, z) \in$ $\mathcal{D}$;

(N3) $\|x+y, z\| \leq\|x, z\|+\|y, z\|$ for $x, y \in X, z \in Y$ such that $(x, z),(y, z) \in$ $\mathcal{D}$.

The set $\mathcal{D}$ is called a 2-normed set.

In particular, if $\mathcal{D}=X \times Y$, the function $\| \cdot$, * $\|$ will be called a generalized 2-norm on $X \times Y$ and the pair $(X \times Y,\|\cdot, \cdot\|)$ a generalized 2-normed space.

Moreover, if $X=Y$, then the generalized 2-normed space will be denoted by $(X,\|\cdot, \cdot\|)$.

Assume that the generalized 2-norm satisfies, in addition, the symmetry condition. Then we will define the 2 -norm as follows:

Definition 1.3. [4] Let $X$ be a real linear space. Denote by $\mathcal{X}$ a nonempty subset of $X \times X$ with the property $\mathcal{X}=\mathcal{X}^{-1}$ and such that the set $\mathcal{X}^{y}=\{x \in X ;(x, y) \in \mathcal{X}\}$ is a linear subspace of $X$, for all $y \in X$.

A function $\|\cdot, \cdot\|: \mathcal{X} \rightarrow[0, \infty)$ satisfying the following conditions:

(S1) $\|x, y\|=\|y, x\|$ for all $(x, y) \in \mathcal{X}$;

(S2) $\|x, \alpha y\|=|\alpha| \cdot\|x, y\|$ for any real number $\alpha$ and all $(x, y) \in \mathcal{X}$;

(S3) $\|x, y+z\| \leq\|x, y\|+\|x, z\|$ for $x, y, z \in X$ such that $(x, y),(x, z) \in \mathcal{X}$; will be called a generalized symmetric 2-norm on $\mathcal{X}$. The set $\mathcal{X}$ is called a symmetric 2-normed set. In particular, if $\mathcal{X}=X \times X$, the function $\|\cdot, \cdot\|$ will be called a generalized symmetric 2-norm on $X$ and the pair $(X,\|\cdot, \cdot\|)$ a generalized symmetric 2-normed space.

In [4], [5], [6], [7] we considered properties of generalized 2-normed spaces and 2-normed sets.

In what follows we shall use the following results:

TheOREm 1.4. [4] Let $(X \times Y,\|\cdot, \cdot\|)$ be a generalized 2-normed space. Then the family $\mathcal{B}$ of all sets defined by

$$
\bigcap_{i=1}^{n}\left\{x \in X ;\left\|x, y_{i}\right\|<\varepsilon\right\},
$$

where $y_{1}, y_{2}, \ldots, y_{n} \in Y, n \in N$ and $\varepsilon>0$, forms a complete system of neighborhoods of zero for a locally convex topology in $X$. 
We will denote it by the symbol $\mathcal{T}(X, Y)$. Similarly, we have the preceding theorem for a topology $\mathcal{T}(Y, X)$ in the space $Y$. In the case when $X=Y$ we will write: $\mathcal{T}_{1}(X)=\mathcal{T}(X, Y)$ and $\mathcal{T}_{2}(X)=\mathcal{T}(Y, X)$.

Let $(X \times Y,\|\cdot, \cdot\|)$ be a generalized 2-normed space and let $\Sigma$ be a directed set. A net $\left\{x_{\sigma} ; \sigma \in \Sigma\right\}$ is convergent to $x_{o} \in X$ in $(X, \mathcal{T}(X, Y))$ if and only if for all $y \in Y$ and $\varepsilon>0$ there exists $\sigma_{o} \in \Sigma$ such that $\left\|x_{\sigma}-x_{o}, y\right\|<\varepsilon$ for all $\sigma \geq \sigma_{o}$. Similarly we have the notion of convergence in $(Y, \mathcal{T}(Y, X))$.

A sequence $\left\{x_{n} ; n \in N\right\} \subset X$ is a Cauchy sequence in $(X, \mathcal{T}(X, Y))$ if and only if for every $y \in Y$ and $\varepsilon>0$ there exists a number $n_{o} \in N$ such that inequality $n, m>n_{o}$ implies $\left\|x_{n}-x_{m}, y\right\|<\varepsilon$. A space $(X, \mathcal{T}(X, Y))$ is called sequentially complete if every Cauchy sequence in $(X, \mathcal{T}(X, Y))$ is convergent in this space. Analogously we have the notion of sequential completeness for the space $(Y, \mathcal{T}(Y, X))$.

EXAmPLE 1.5. [4] Let $X$ be a real linear space which have two norms (seminorms) $\|\cdot\|_{1},\|\cdot\|_{2}$. Then $(X,\|\cdot, \cdot\|)$ is a generalized 2-normed space with the 2-norm defined by the formula

$$
\|x, y\|=\|x\|_{1} \cdot\|y\|_{2} \text { for each } x, y \in X .
$$

Let us remark that topologies generated by these norms $\|\cdot\|_{1}$ and $\|\cdot\|_{2}$ coincide with the topologies $\mathcal{T}_{1}(X)$ and $\mathcal{T}_{2}(X)$ given in Theorem 1.4.

Example 1.6. In Example 1.5 we can get $\|\cdot\|_{1}=\|\cdot\|_{2}$. Then $(X,\|\cdot, \cdot\|)$ is a generalized symmetric 2 -normed space with the symmetric 2-norm defined by the formula

$$
\|x, y\|=\|x\| \cdot\|y\| \text { for each } x, y \in X .
$$

Let us remark that a symmetric 2-normed space need not be a 2-normed space in the sense of Gähler. For instance given in Example 1.6 $x \neq \theta, y=$ $k x, k \neq 0$ we obtain

$$
\|x, y\|=\|x, k x\|=|k| \cdot\|x, x\|=|k| \cdot\|x\|^{2}>0,
$$

but in spite of this $x$ and $y$ are linearly dependent. The 2-normed space from Example 1.6 is not a 2-normed space in the sense of Definition 1.1.

It is easy to see that if $(X,\|\cdot\|)$ is a normed space, $\mathcal{T}_{1}$-the topology generated by this norm and $\mathcal{T}_{2}$-the topology generated by the 2-norm defined by the formula (1.1), then $\mathcal{T}_{1}=\mathcal{T}_{2}$. Moreover a sequence $\left\{x_{n} ; n \in N\right\}$ is a Cauchy sequence in $(X,\|\cdot\|)$ if and only if it is a Cauchy sequence in $(X,\|\cdot, \cdot\|)$ with the 2-norm defined in Example 1.6.

Thus the following theorem follows.

Theorem 1.7. A normed space $(X,\|\cdot\|)$ is a Banach space if and only if the symmetric 2-normed space with the 2-norm defined by (1.1) is sequentially complete. 


\section{The SPACE of All Bounded 2-Linear operators}

In [8] A. G. White defined and considered the properties of bounded 2linear functionals from $B \times B$, where $B$ denotes a 2-normed space in the sense of Gähler. He proved that the set of all bounded 2-linear functionals is a Banach space.

S. S. Kim, Y. J. Cho and A. G. White in [3] and A. Khan in [2] gave the properties of bounded operators from $X \times X$ with values in a normed space $Y$, where $X$ denotes a 2-normed space in the sense of Gähler. They showed that the set $B(X \times X, Y)$ of all bounded operators from $X \times X$ into $Y$ is a seminormed space. Moreover, if $Y$ is a Banach space, then $B(X \times X, Y)$ is a complete space.

In this section we will consider bounded 2-linear operators defined on a 2 -normed set into a normed space. We will show, like in the above mentioned papers, that the space of these operators is a Banach space. We will prove that under some additional conditions it is a symmetric 2-normed space.

Let us consider a real linear space $X$. Let $\mathcal{D} \subset X \times X$ be a 2 -normed set, $Y$ a normed space.

DeFinition 2.1. An operator $F: \mathcal{D} \rightarrow Y$ is said to be 2-linear if it satisfies the following conditions:

1. $F(a+c, b+d)=F(a, b)+F(a, d)+F(c, b)+F(c, d)$ for $a, b, c, d \in X$ such that $a, c \in \mathcal{D}^{b} \cap \mathcal{D}^{d}$.

2. $F(\alpha a, \beta b)=\alpha \cdot \beta \cdot F(a, b)$ for $\alpha, \beta \in \mathbb{R},(a, b) \in \mathcal{D}$.

Definition 2.2. A 2-normed operator $F$ is said to be bounded if there is a positive number $K$ such that

$$
\|F(a, b)\| \leq K \cdot\|a, b\| \text { for all }(a, b) \in \mathcal{D} .
$$

DEFINITION 2.3. If $F$ is a bounded operator, then the following number

$$
\|F\|=\inf \{K>0 ;\|F(a, b)\| \leq K \cdot\|a, b\| \text { for }(a, b) \in \mathcal{D}\}
$$

will be called the norm of the 2-linear operator $F$.

Example 2.4. Let $(X,(\cdot \mid \cdot))$ be a real inner product space. Then $X$ is a generalized symmetric 2-normed space with the 2-norm defined as follows:

$$
\|x, y\|=|(x \mid y)| \text { for all } x, y \in X .
$$

This 2-norm generates a weak topology in the Hilbert space (see Example 1.5 in [4]). An operator $F: X \times X \rightarrow \mathbb{R}$ defined by the formula

$$
F(a, b)=(a \mid b) \text { for } a, b \in X
$$

is 2-linear and bounded. Moreover $\|F\|=1$.

In the next theorem we will give properties of the above mentioned notions. 
TheOREM 2.5. Let $F$ be a bounded 2-linear operator. Then:

(a) $\|F\| \leq K$ for $K \in \mathcal{P}^{(F)}=\left\{K^{\prime}>0 ;\|F(a, b)\| \leq K^{\prime} \cdot\|a, b\|\right.$ for $(a, b) \in \mathcal{D}\}$

(b) $\|F(a, b)\| \leq\|F\| \cdot\|a, b\|$ for each $(a, b) \in \mathcal{D}$;

(c)

$$
\begin{aligned}
\|F\| & =\sup \{\|F(a, b)\| ;(a, b) \in \mathcal{D},\|a, b\|=1\} \\
& =\sup \{\|F(a, b)\| ;(a, b) \in \mathcal{D},\|a, b\| \leq 1\} \\
& =\sup \left\{\frac{\|F(a, b)\|}{\|a, b\|} ;(a, b) \in \mathcal{D},\|a, b\| \neq 0\right\} .
\end{aligned}
$$

Proof. The condition (a) follows from the Definition 2.3.

(b) Because the operator $F$ is bounded, then there exists $K>0$ such that

$$
\|F(a, b)\| \leq K \cdot\|a, b\| \text { for }(a, b) \in \mathcal{D} .
$$

Thus $\|F(a, b)\| \leq \inf _{K^{\prime} \in \mathcal{P}^{(F)}} K^{\prime} \cdot\|a, b\|$, i.e.

$$
\|F(a, b)\| \leq\|F\| \cdot\|a, b\| .
$$

(c) By (b), $\sup \left\{\frac{\|F(a, b)\|}{\|a, b\|} ;(a, b) \in \mathcal{D},\|a, b\| \neq 0\right\} \leq\|F\|$.

Let $A=\sup \{\|F(a, b)\| ;(a, b) \in \mathcal{D},\|a, b\|=1\}$. Then

$$
\begin{aligned}
A & =\sup \left\{\frac{\|F(a, b)\|}{\|a, b\|} ;(a, b) \in \mathcal{D},\|a, b\|=1\right\} \\
& \leq \sup \left\{\frac{\|F(a, b)\|}{\|a, b\|} ;(a, b) \in \mathcal{D},\|a, b\| \leq 1\right\} \\
& \leq \sup \left\{\frac{\|F(a, b)\|}{\|a, b\|} ;(a, b) \in \mathcal{D},\|a, b\| \neq 0\right\} \\
& \leq\|F\| .
\end{aligned}
$$

Moreover

$$
A \leq \sup \{\|F(a, b)\| ;(a, b) \in \mathcal{D},\|a, b\| \leq 1\} .
$$

Let $(a, b) \in \mathcal{D}$ be such that $\|a, b\| \neq 0$. Because $\left\|\frac{a}{\|a, b\|}, b\right\|=1$, then $\left\|F\left(\frac{a}{\|a, b\|}, b\right)\right\| \leq A$. And further by virtue of the equalities

$$
\left\|F\left(\frac{a}{\|a, b\|}, b\right)\right\|=\left\|\frac{1}{\|a, b\|} \cdot F(a, b)\right\|=\frac{1}{\|a, b\|} \cdot\|F(a, b)\|
$$

we obtain $\|F(a, b)\| \leq A \cdot\|a, b\|$. On the other hand, if $(a, b) \in \mathcal{D}$ and $\|a, b\|=$ 0 , then $0 \leq\|F(a, b)\| \leq\|F\| \cdot\|a, b\|=0$, i.e. $\|F(a, b)\|=0=A \cdot\|a, b\|$. 
Consequently $\|F(a, b)\| \leq A \cdot\|a, b\|$ for all $(a, b) \in \mathcal{D}$, which means that $A \in \mathcal{P}^{(F)}$. By virtue of (a) we obtain

$$
\|F\| \leq A \text {. }
$$

The conditions (2.1) and (2.3) imply

$$
\begin{aligned}
\|F\| & =\sup \{\|F(a, b)\| ;(a, b) \in \mathcal{D},\|a, b\|=1\} \\
& =\sup \left\{\frac{\|F(a, b)\|}{\|a, b\|} ;(a, b) \in \mathcal{D},\|a, b\| \neq 0\right\} .
\end{aligned}
$$

From (b) we have $\sup \{\|F(a, b)\| ;(a, b) \in \mathcal{D},\|a, b\| \leq 1\} \leq\|F\|$, which with (2.2) gives the equality $\|F\|=\sup \{\|F(a, b)\| ;(a, b) \in \mathcal{D},\|a, b\| \leq 1\}$, and the proof is completed.

Definition 2.6. Let $\mathcal{D} \subset X \times X$ be a 2-normed set and $Y$ a normed space. Denote by $L_{2}(\mathcal{D}, Y)$ the set of all bounded 2-linear operators from $\mathcal{D}$ into $Y$.

In particular, we will write $L_{2}(X, Y)$, if $X$ is a generalized 2-normed space and $\mathcal{D}=X \times X$.

Let $F, G \in L_{2}(\mathcal{D}, Y)$ and define

1. $(F+G)(a, b)=F(a, b)+G(a, b)$ for all $(a, b) \in \mathcal{D}$;

2. $(\alpha \cdot F)(a, b)=\alpha \cdot F(a, b)$ for $\alpha \in \mathbb{R},(a, b) \in \mathcal{D}$.

THEOREM 2.7. If $\mathcal{D}$ is a 2-normed set and $Y$ a normed space, then the set $L_{2}(\mathcal{D}, Y)$ is a normed space with the norm $\|\cdot\|$ defined in Definition 2.3.

Proof. Let us take $F, G \in L_{2}(\mathcal{D}, Y), \alpha, \beta \in \mathcal{R}$ and $a, b, c, d \in X$ such that $a, c \in \mathcal{D}^{b} \cap \mathcal{D}^{d}$. For $F+G$ we obtain:

$$
\begin{aligned}
& (F+G)(a+c, b+d)= \\
& =(F+G)(a, b)+(F+G)(a, d)+(F+G)(c, b)+(F+G)(c, d) ; \\
& (F+G)(\alpha a, \beta b)=\alpha \beta \cdot(F+G)(a, b) .
\end{aligned}
$$

Moreover by virtue of the condition (b) of Theorem 2.5 we have

$$
\begin{aligned}
\|(F+G)(a, b)\| & =\|F(a, b)+G(a, b)\| \\
& \leq\|F(a, b)\|+\|G(a, b)\| \leq\|F\| \cdot\|a, b\|+\|G\| \cdot\|a, b\| \\
& =(\|F\|+\|G\|) \cdot\|a, b\| .
\end{aligned}
$$

Thus $F+G \in L_{2}(\mathcal{D}, Y)$.

Analogously we show that $\alpha \cdot F \in L_{2}(\mathcal{D}, Y)$ and

$$
\|(\alpha \cdot F)(a, b)\|=\|\alpha \cdot F(a, b)\| \leq|\alpha| \cdot\|F\| \cdot\|a, b\| .
$$

Moreover it is easy to prove that the set $L_{2}(\mathcal{D}, Y)$ is a real linear space.

Now we will show that the function $\|\cdot\|: L_{2}(\mathcal{D}, Y) \rightarrow[0, \infty)$ given in Definition 2.3 satisfies all conditions of a norm. 
If $\|F\|=0$, then $\|F(a, b)\|=0$ for all $(a, b) \in \mathcal{D}$. Thus $F(a, b)=0$ for every $(a, b) \in \mathcal{D}$. Conversely, if $F$ is a zero operator, then

$$
\|F\|=\sup \{\|F(a, b)\| ;(a, b) \in \mathcal{D},\|a, b\|=1\}=0 .
$$

As a consequence we have the condition

$$
\|F\|=0 \text { if and only if } F=0 .
$$

From (2.7) we have $|\alpha| \cdot\|F\| \in \mathcal{P}^{(\alpha F)}$, which with Theorem 2.5 (a) implies the inequality $\|\alpha \cdot F\| \leq|\alpha| \cdot\|F\|$. Assume $\alpha \neq 0$. Then

$$
\|F\|=\left\|\frac{1}{\alpha} \cdot \alpha \cdot F\right\| \leq \frac{1}{|\alpha|} \cdot\|\alpha F\|,
$$

i.e. $|\alpha| \cdot\|F\| \leq\|\alpha \cdot F\|$; thus $|\alpha| \cdot\|F\|=\|\alpha \cdot F\|$.

For $\alpha=0$ the equality $\|\alpha \cdot F\|=|\alpha| \cdot\|F\|$ is obvious. Therefore $\|\alpha \cdot F\|=|\alpha| \cdot\|F\|$ for $\alpha \in \mathbb{R}$.

The condition (2.6) implies $\|F\|+\|G\| \in \mathcal{P}^{(F+G)}$. Hence and from Theorem 2.5(a) we have $\|F+G\| \leq\|F\|+\|G\|$. This completes the proof.

THEOREM 2.8. If $\mathcal{D}$ is a 2-normed set and $Y$ is a Banach space, then $L_{2}(\mathcal{D}, Y)$ is a Banach space.

Proof. According to Theorem 2.7, $L_{2}(\mathcal{D}, Y)$ is a normed space.

Let $\left\{F_{n} ; n \in N\right\}$ be a Cauchy sequence in $L_{2}(\mathcal{D}, Y)$. Then

$$
\lim _{n, m \rightarrow \infty}\left\|F_{n}-F_{m}\right\|=0
$$

and for every $(a, b) \in \mathcal{D}$ the following inequality

$$
\left\|F_{n}(a, b)-F_{m}(a, b)\right\|=\left\|\left(F_{n}-F_{m}\right)(a, b)\right\| \leq\left\|F_{n}-F_{m}\right\| \cdot\|a, b\|
$$

is true. Thus $\left\{F_{n}(a, b) ; n \in N\right\}$ is a Cauchy sequence in $Y$ for every $(a, b) \in \mathcal{D}$. Because $Y$ is complete, the sequence $\left\{F_{n}(a, b) ; n \in N\right\}$ is convergent for every $(a, b) \in \mathcal{D}$. Let us denote

$$
F(a, b)=\lim _{n \rightarrow \infty} F_{n}(a, b) .
$$

We shall show that $F \in L_{2}(\mathcal{D}, Y)$.

For $a, b, c, d \in X$ such that $a, c \in \mathcal{D}^{b} \cap \mathcal{D}^{d}$ we have

$$
\begin{aligned}
F(a & +c, b+d)=\lim _{n \rightarrow \infty} F_{n}(a+c, b+d) \\
& =\lim _{n \rightarrow \infty} F_{n}(a, b)+\lim _{n \rightarrow \infty} F_{n}(a, d)+\lim _{n \rightarrow \infty} F_{n}(c, b)+\lim _{n \rightarrow \infty} F_{n}(c, d) \\
& =F(a, b)+F(a, d)+F(c, b)+F(c, d) .
\end{aligned}
$$


Moreover for $\alpha, \beta \in \mathbb{R}$ and $(a, b) \in \mathcal{D}$ we have:

$$
\begin{aligned}
F(\alpha a, \beta b) & =\lim _{n \rightarrow \infty} F_{n}(\alpha a, \beta b) \\
& =\lim _{n \rightarrow \infty} \alpha \beta \cdot F_{n}(a, b) \\
& =\alpha \beta \cdot \lim _{n \rightarrow \infty} F_{n}(a, b) \\
& =\alpha \beta \cdot F(a, b) .
\end{aligned}
$$

Thus $F$ is a 2-linear operator.

The inequality

$$
\left|\left\|F_{n}\right\|-\left\|F_{m}\right\|\right| \leq\left\|F_{n}-F_{m}\right\|
$$

implies that $\left\{\left\|F_{n}\right\| ; n \in N\right\}$ is a Cauchy sequence in $\mathbb{R}$. As a consequence this sequence is bounded, that is, there exists $K>0$ such that $\left\|F_{n}\right\| \leq K$ for all $n \in N$. Using this result we get

$$
\begin{aligned}
\|F(a, b)\| & \leq\left\|F_{n}(a, b)\right\|+\left\|F(a, b)-F_{n}(a, b)\right\| \\
& \leq\left\|F_{n}\right\| \cdot\|a, b\|+\left\|F(a, b)-F_{n}(a, b)\right\| \\
& \leq K \cdot\|a, b\|+\left\|F_{n}(a, b)-F(a, b)\right\| .
\end{aligned}
$$

Letting $n \rightarrow \infty$ we obtain $\|F(a, b)\| \leq K \cdot\|a, b\|$ for every $(a, b) \in \mathcal{D}$, which means that $F$ is bounded. So we have shown that $F \in L_{2}(\mathcal{D}, Y)$.

Now let us suppose that $(a, b) \in \mathcal{D}$ and $\|a, b\| \neq 0$. Let $\varepsilon>0$. Because $\left\{F_{n} ; n \in N\right\}$ is a Cauchy sequence, there exists $n_{o} \in N$ such that

$$
\left\|F_{n}-F_{m}\right\|<\frac{\varepsilon}{4} \text { for all } n, m \geq n_{o} .
$$

Thus $\left\|F_{n}(a, b)-F_{m}(a, b)\right\| \leq\left\|F_{n}-F_{m}\right\| \cdot\|a, b\|<\frac{\varepsilon}{4} \cdot\|a, b\|$ for all $n, m \geq n_{o}$. The equality

$$
F(a, b)=\lim _{n \rightarrow \infty} F_{n}(a, b)
$$

implies that there exists $n_{1}=n_{1}(a, b) \geq n_{o}$ such that

$$
\left\|F_{n_{1}}(a, b)-F(a, b)\right\|<\frac{\varepsilon}{4} \cdot\|a, b\| .
$$

As a consequence we obtain

$$
\begin{aligned}
\left\|F_{n}(a, b)-F(a, b)\right\| & \leq\left\|F_{n}(a, b)-F_{n_{1}}(a, b)\right\|+\left\|F_{n_{1}}(a, b)-F(a, b)\right\| \\
& <\frac{\varepsilon}{2} \cdot\|a, b\|
\end{aligned}
$$

for $n \geq n_{o},(a, b) \in \mathcal{D}$ and $\|a, b\| \neq 0$. If $\|a, b\|=0$, then $F_{n}(a, b)=0=$ $F(a, b)$, so $\left\|F_{n}(a, b)-F(a, b)\right\|=\frac{\varepsilon}{2} \cdot\|a, b\|$. Thus $\left\|F_{n}(a, b)-F(a, b)\right\| \leq \frac{\varepsilon}{2} \cdot\|a, b\|$ for all $n \geq n_{o},(a, b) \in \mathcal{D}$, i.e.

$$
\frac{\varepsilon}{2} \in \mathcal{P}\left(F_{n}-F\right) \quad \text { for } n \geq n_{o}
$$


Therefore $\left\|F_{n}-F\right\| \leq \frac{\varepsilon}{2}<\varepsilon$ for $n \geq n_{o}$, which means that the sequence $\left\{F_{n} ; n \in N\right\}$ is convergent to $F$ in $L_{2}(\mathcal{D}, Y)$. Hence we have shown that $L_{2}(\mathcal{D}, Y)$ is a Banach space, which finishes the proof.

From Theorem 2.8 and Theorem 1.7 the following corollary follows.

Corollary 2.9. If $\mathcal{X}$ is a symmetric 2-normed set and $Y$ is a Banach space, then $L_{2}(\mathcal{X}, Y)$ is a symmetric sequentially complete 2-normed space with the 2-norm defined as follows:

$$
\|F, G\|=\|F\| \cdot\|G\| \text { for } F, G \in L_{2}(\mathcal{X}, Y) .
$$

\section{Banach-Steinhaus Theorems for Bounded 2-Linear operators}

In this section we will consider properties of sequences of operators from $L_{2}(\mathcal{D}, Y)$. We will formulate Banach-Steinhaus Theorems for a family of these operators.

Proposition 3.1. Let $\mathcal{D}$ be a 2-normed set, $Y$ a normed space and $\left\{F_{n} ; n \in N\right\} \subset L_{2}(\mathcal{D}, Y)$. If the sequence of norms $\left\{\left\|F_{n}\right\| ; n \in N\right\}$ is bounded, then for each $(x, y) \in \mathcal{D}$ the sequence of norms $\left\{\left\|F_{n}(x, y)\right\| ; n \in N\right\}$ is bounded.

Proof. From the assumption it follows that there exists a positive number $M$ such that $\left\|F_{n}\right\| \leq M$ for each $n \in N$. Thus for $(x, y) \in \mathcal{D}$ we obtain

$$
\left\|F_{n}(x, y)\right\| \leq\left\|F_{n}\right\| \cdot\|x, y\| \leq M \cdot\|x, y\| \text { for each } n \in N .
$$

THEOREM 3.2. Let $X$ be a generalized 2-normed space and $Y$ a normed space. If $\left\{F_{n} ; n \in N\right\} \subset L_{2}(X, Y)$ is pointwise convergent to $F$ and the sequence of norms $\left\{\left\|F_{n}\right\| ; n \in N\right\}$ is bounded, then $F \in L_{2}(X, Y)$.

Proof. For all $x, y \in X$ we have

$$
F(x, y)=\lim _{n \rightarrow \infty} F_{n}(x, y) .
$$

Thus the operator $F$ is a 2-linear operator.

Because the sequence of norms $\left\{\left\|F_{n}\right\| ; n \in N\right\}$ is bounded, then there exists $M>0$ such that $\left\|F_{n}\right\| \leq M$ for all $n \in N$. Thus $\left\|F_{n}(x, y)\right\| \leq$ $\left\|F_{n}\right\| \cdot\|x, y\| \leq M \cdot\|x, y\|$. Let us take $x, y \in X$. Then

$$
\begin{aligned}
\|F(x, y)\| & \leq\left\|F_{n}(x, y)-F(x, y)\right\|+\left\|F_{n}(x, y)\right\| \leq \\
& \leq\left\|F_{n}(x, y)-F(x, y)\right\|+M \cdot\|x, y\| .
\end{aligned}
$$

By letting $n \rightarrow \infty$ we obtain $\|F(x, y)\| \leq M \cdot\|x, y\|$ for each $x, y \in X$. This gives that $F$ is bounded. As a consequence we have shown that $F \in L_{2}(X, Y)$. 
TheOREm 3.3. Let $Y$ be a Banach space, $(X,\|\cdot, \cdot\|)$ a generalized 2normed space and let $A$ be a linearly dense set in the spaces $\left(X, \mathcal{T}_{1}(X)\right)$ and $\left(X, \mathcal{T}_{2}(X)\right)$. If a sequence $\left\{F_{n} ; n \in N\right\} \subset L_{2}(X, Y)$ is pointwise convergent on the set $A$ and the sequence of norms $\left\{\left\|F_{n}\right\| ; n \in N\right\}$ is bounded, then the sequence $\left\{F_{n}(x, y) ; n \in N\right\}$ is convergent in $Y$ for each $x, y \in X$.

Proof. Let $X_{O}$ be the linear subspace of $X$ generated by $A$. We will consider $X_{o}$ as a 2 -normed space with the same 2 -norm induced by that of $X$. Let $x, y \in X_{o}$. Then $x=a_{1} x_{1}+\cdots+a_{k} x_{k}, y=b_{1} y_{1}+\cdots+b_{t} y_{t}$, where $a_{i}, b_{j} \in \mathbb{R}$, $x_{i}, y_{j} \in A, i=1,2, \ldots, k, j=1,2, \ldots, t ; k, t \in N$, and

$$
F_{n}(x, y)=\sum_{i=1}^{k} \sum_{j=1}^{t} a_{i} b_{j} \cdot F_{n}\left(x_{i}, y_{j}\right) .
$$

Because the sequence $\left\{F_{n}\left(x_{i}, y_{j}\right) ; n \in N\right\}$ is convergent for all $x_{i}, y_{j} \in A$, then $\left\{F_{n}(x, y) ; n \in N\right\}$ is convergent in $X_{o}$.

Let $\left\|F_{n}\right\| \leq M$ for every $n \in N$. Let us take a number $\varepsilon>0$ and $x, y \in X$. Since $X_{o}$ is a dense set in $\left(X, \mathcal{T}_{1}(X)\right)$ we can choose $x_{o} \in X_{o}$ such that

$$
\left\|x-x_{o}, y\right\|<\frac{\varepsilon}{6 M} \text {. }
$$

Moreover there exists $y_{o} \in X_{o}$ with the property

$$
\left\|x_{o}, y-y_{o}\right\|<\frac{\varepsilon}{6 M}
$$

because $X_{o}$ is also a dense set in $\left(X, \mathcal{T}_{2}(X)\right)$.

The sequence $\left\{F_{n}\left(x_{o}, y_{o}\right) ; n \in N\right\}$ is convergent, so it is a Cauchy sequence in $Y$. Therefore there exists a number $n_{o} \in N$ such that

$$
\left\|F_{n}\left(x_{o}, y_{o}\right)-F_{m}\left(x_{o}, y_{o}\right)\right\|<\frac{\varepsilon}{3} \text { for each } n, m \geq n_{o} .
$$

As a consequence we obtain

$$
\begin{aligned}
\left\|F_{n}(x, y)-F_{m}(x, y)\right\|= & \left\|F_{n}\left(x-x_{o}+x_{o}, y\right)-F_{m}\left(x-x_{o}+x_{o}, y\right)\right\| \\
\leq & \left\|F_{n}\left(x-x_{o}, y\right)\right\|+\left\|F_{m}\left(x-x_{o}, y\right)\right\| \\
& +\left\|F_{n}\left(x_{o}, y\right)-F_{m}\left(x_{o}, y\right)\right\| \\
\leq & \left\|F_{n}\left(x-x_{o}, y\right)\right\|+\left\|F_{m}\left(x-x_{o}, y\right)\right\| \\
& +\left\|F_{n}\left(x_{o}, y-y_{o}\right)\right\|+\left\|F_{m}\left(x_{o}, y-y_{o}\right)\right\| \\
& +\left\|F_{n}\left(x_{o}, y_{o}\right)-F_{m}\left(x_{o}, y_{o}\right)\right\| \\
\leq & \left\|F_{n}\right\| \cdot\left\|x-x_{o}, y\right\|+\left\|F_{m}\right\| \cdot\left\|x-x_{o}, y\right\| \\
& +\left\|F_{n}\right\| \cdot\left\|x_{o}, y-y_{o}\right\|+\left\|F_{m}\right\| \cdot\left\|x_{o}, y-y_{o}\right\|+\frac{\varepsilon}{3} \\
\leq & 2 M \cdot\left\|x-x_{o}, y\right\|+2 M \cdot\left\|x_{o}, y-y_{o}\right\|+\frac{\varepsilon}{3}<\varepsilon
\end{aligned}
$$


for $n, m \geq n_{o}$. Hence we have shown that $\left\{F_{n}(x, y) ; n \in N\right\}$ is a Cauchy sequence in $Y$ for each $x, y \in X$. Because $Y$ is complete, then the sequence $\left\{F_{n}(x, y) ; n \in N\right\}$ is convergent in $Y$, which finishes the proof.

Theorem 3.4. Let $(X,\|\cdot, \cdot\|)$ be a generalized 2-normed space and $Y$ a Banach space. If a sequence $\left\{F_{n} ; n \in N\right\} \subset L_{2}(X, Y)$ is pointwise convergent to $F \in L_{2}(X, Y)$ on a linearly dense set $A$ in the spaces $\left(X, \mathcal{T}_{1}(X)\right)$ and $\left(X, \mathcal{T}_{2}(X)\right)$ and the sequence of norms $\left\{\left\|F_{n}\right\| ; n \in N\right\}$ is bounded, then $\left\{F_{n} ; n \in N\right\}$ is pointwise convergent to $F$ and the inequality $\|F\| \leq \sup _{n}\left\|F_{n}\right\|$ holds.

Proof. It follows from Theorem 3.3 that the sequence $\left\{F_{n}(x, y) ; n \in N\right\}$ is convergent in $Y$ for each $x, y \in X$. Let us denote

$$
H(x, y)=\lim _{n \rightarrow \infty} F_{n}(x, y) \text { for every } x, y \in X .
$$

We must show that $H(x, y)=F(x, y)$ for all $x, y \in X$. Using Theorem 3.2 we see that $H \in L_{2}(X, Y)$. From assumption it follows that $H(x, y)=F(x, y)$ for all $x, y \in A$, i.e. $(H-F)(x, y)=0$ for $x, y \in A$. Because $L_{2}(X, Y)$ is a linear space, then $H-F \in L_{2}(X, Y)$. As a consequence $H-F$ is an 2-linear operator and $(H-F)(x, y)=0$ for $x, y \in X_{o}$, where $X_{o}$ denote the set of all linear combinations of elements from $A$. Moreover $H-F$ is bounded, thus there exists $K>0$ such that $\|(H-F)(x, y)\| \leq K \cdot\|x, y\|$ for every $x, y \in X$.

Let $\varepsilon>0, x, y \in X$. Since the set $X_{o}$ is dense in $\left(X, \mathcal{T}_{1}(X)\right)$ we can choose $x_{o} \in X_{o}$ such that

$$
\left\|x-x_{o}, y\right\|<\frac{\varepsilon}{2 K} .
$$

There exists $y_{o} \in X_{o}$ with the property

$$
\left\|x_{o}, y-y_{o}\right\|<\frac{\varepsilon}{2 K}
$$

because $X_{o}$ is also dense in $\left(X, \mathcal{T}_{2}(X)\right)$. Then we have

$$
\begin{aligned}
0 & \leq\|(H-F)(x, y)\|=\left\|(H-F)\left(x-x_{o}+x_{o}, y\right)\right\| \\
& =\left\|(H-F)\left(x-x_{o}, y\right)+(H-F)\left(x_{o}, y\right)\right\| \\
& =\left\|(H-F)\left(x-x_{o}, y\right)+(H-F)\left(x_{o}, y-y_{o}+y_{o}\right)\right\| \\
& =\left\|(H-F)\left(x-x_{o}, y\right)+(H-F)\left(x_{o}, y-y_{o}\right)+(H-F)\left(x_{o}, y_{o}\right)\right\| \\
& =\left\|(H-F)\left(x-x_{o}, y\right)+(H-F)\left(x_{o}, y-y_{o}\right)\right\| \\
& \leq\left\|(H-F)\left(x-x_{o}, y\right)\right\|+\left\|(H-F)\left(x_{o}, y-y_{o}\right)\right\| \\
& \leq K \cdot\left\|x-x_{o}, y\right\|+K \cdot\left\|x_{o}, y-y_{o}\right\|<\varepsilon .
\end{aligned}
$$

This gives $\|(H-F)(x, y)\|=0$ for each $x, y \in X$, i.e. $H(x, y)=F(x, y)$ for every $x, y \in X$. 
Let us denote $M=\sup _{n}\left\|F_{n}\right\|$. Then for every $n \in N$ and $x, y \in X$ such that $\|x, y\| \leq 1$ we have

$$
\left\|F_{n}(x, y)\right\| \leq\left\|F_{n}\right\| \cdot\|x, y\| \leq M
$$

Thus

$$
\begin{aligned}
\|F(x, y)\| & =\left\|F(x, y)-F_{n}(x, y)+F_{n}(x, y)\right\| \\
& \leq\left\|F(x, y)-F_{n}(x, y)\right\|+\left\|F_{n}(x, y)\right\| \\
& \leq\left\|F(x, y)-F_{n}(x, y)\right\|+M .
\end{aligned}
$$

By letting $n \rightarrow \infty$ we obtain $\|F(x, y)\| \leq M$ for $x, y \in X$ such that $\|x, y\| \leq 1$. This implies $\|F\|=\sup \{\|F(x, y)\| ; x, y \in X,\|x, y\| \leq 1\} \leq M$, which finishes the proof.

\section{REFERENCES}

[1] S. Gähler, Lineare 2-normierte Räume, Math. Nachr. 28 (1964), 1-43.

[2] A. Khan, Lipschitz 2-operators, Math. Seminar Notes, Kobe Univ. 10 (1982), 367-372.

[3] S.S. Kim, Y.J. Cho and A. White, Linear operators on linear 2-normed spaces, Glasnik Mat. 27(47) (1992), 63-70.

[4] Z. Lewandowska, Linear operators on generalized 2-normed spaces, Bull. Math. Soc. Sc. Math. Roumanie 42 (1999), 353-368.

[5] Z. Lewandowska, Generalized 2-normed spaces, Słupskie Prace MatematycznoFizyczne 1 (2001), 33-40.

[6] Z. Lewandowska, On 2-normed sets, Glasnik Mat. 38 (2003), 99-110.

[7] Z. Lewandowska, Banach-Steinhaus Theorems for bounded linear operators with values in a generalized 2-normed space, Glasnik Mat. 38 (2003), 329-340.

[8] A. White, 2-Banach spaces, Math. Nachr. 42 (1969), 43-60.

Z. Lewandowska

Department of Mathematics

Pedagogical University

Arciszewskiego $22 \mathrm{~b}$

Pl-76-200 Slupsk

Poland

E-mail: lewandow@pap.edu.pl \& Lewandowscy@rene.com.pl

Received: 1.10.2003. 